\title{
NUTRIÇÃO PARENTERAL - PRÍNCIPIOS GERAIS, FORMULÁRIOS DE PRESCRIÇÃO E MONITORIZAÇÃO
}

\author{
PARENTERAL NUTRITION SUPPORT - PRESCRIPTIONS AND FOLLOW UP
}

Júlio Sérgio Marchini ${ }^{1}$, Nelson Okano², Palmira Cupo ${ }^{3}$, Nilva Maria Rodrigues R. da Silva Passos ${ }^{4}$, Luiz Maçao Sakamoto ${ }^{4}$ \& Anibal Basile-Filho ${ }^{5}$

\begin{abstract}
1'Docente do Departamento de Clínica Médica — Divisão de Nutrição Clínica, ${ }^{2,5}$ Docente do Departamento de Cirurgia, Ortopedia e Traumatologia, ${ }^{3}$ Docente do Departamento de Puericultura e Pediatria, ${ }^{4}$ Farmacêutico da Divisão de Assistência Farmacêutica do Hospital das Clínicas de Ribeirão Preto; Faculdade de Medicina de Ribeirão Preto da Universidade de São Paulo.

CorRespondÊncia: J. Sérgio Marchini - Departamento de Clínica Médica da FMRP-USP — Hospital das Clínicas - 6 Andar-Campus USP -14048-900 — Ribeirão Preto - SP. Telefone (016) 6330436 - E-mail: jsmarchi@fmrp.usp.br
\end{abstract}

MARCHINI JS et al. Nutrição parenteral — princípios gerais, formulários de prescrição e monitorização. Medicina, Ribeirão Preto, 31: 62-72, jan./mar. 1998.

RESUMO: O suporte nutricional, na terapêutica de pacientes hospitalizados, requer o desenvolvimento de princípios que determinarão a melhor assistência nutricional, associada ao menor custo do procedimento. Reconhece-se, atualmente, o impacto causado pela formação de equipes ou comissões multidisciplinares de suporte nutricional parenteral, formadas por médicos, enfermeiros e farmacêuticos, sobre a racionalização da terapêutica nutricional, como a escolha de nutrientes específicos e a padronização das formulações nutritivas. $O$ objetivo deste trabalho se relaciona com o uso de nutrição parenteral total, no Hospital das Clínicas de Ribeirão Preto, incluindo indicações, formulações, efeitos colaterais e benefícios. Paralelamente, é apresentada a padronização das formulações nutritivas parenterais, efetuada pela Comissão de Nutrição Parenteral do Hospital das Clínicas de Ribeirão Preto, a ser utilizada nos pacientes hospitalizados, que necessitem de terapia nutricional parenteral.

UNITERMOS: Nutrição Parenteral Total. Formulários Comissão.

\section{INTRODUÇÃO}

Desde a introdução de uma técnica coerente de suporte nutricional, via parenteral, proposta por

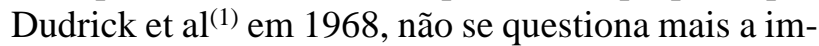
portância da nutrição na recuperação de pacientes clínicos ou cirúrgicos, hospitalizados. Na verdade, as conseqüências da desnutrição de pacientes hospitalizados têm sido objeto de estudo há muito tempo. Assim, sabe-se que aproximadamente $50 \%$ desses pacientes são desnutridos, não importando o tamanho/tipo do hospital, a idade, a doença de base ou a classifica- ção sócio-econômica dos mesmos ${ }^{(3)}$. Tornou-se evidente, também, que a desnutrição está ligada a um aumento de complicações no pós-operatório, como retardo na cicatrização das feridas e anastomoses intestinais, incidência aumentada de infecções por depressão do sistema imunológico e, conseqüentemente, prolongamento no tempo de hospitalização e redução nas chances de sobrevida.

No entanto, a rápida proliferação das técnicas de suporte nutricional parenteral foi responsável pela sua utilização em larga escala, algumas vezes, de forma abusiva, tornando o procedimento extremamente 
oneroso para os hospitais. Reconhece-se, atualmente, o impacto causado pela formação de equipes ou comissões multidisciplinares de padronização de suporte nutricional sobre a redução dos gastos hospitalares, sua racionalização, a padronização dos nutrientes administrados, o seu preparo e controle de qualidade.

\section{INDICAÇÃO}

O suporte nutricional via parenteral está indicado sempre que o paciente está impossibilitado de usar a via enteral por um tempo predefinido. Um outro fator a ser considerado é se o seu uso vai beneficiar o paciente. Assim, por exemplo, dentro do contingente de pacientes desnutridos, internados, nem sempre os pa cientes terminais vão se beneficiar dessa terapêutica.

A distinção do paciente que vai se beneficiar envolve aspectos relacionados com a doença de base e a experiência clínica da equipe de suporte nutricional. Nem sempre esta é uma decisão fácil de ser tomada. O primeiro passo a ser considerado é se o processo mórbido em si vai ser influenciado pelo suporte nutricional parenteral, se a doença ou o tratamento vai piorar o apetite, alterar a digestão/absorção e qual a sua duração. Desde que a prevenção da desnutrição é um procedimento considerado mais fácil do que o tratamento em si, sempre que possível deve-se prevenir o aparecimento da desnutrição intra-hospitalar. Em geral, pacientes com perda de massa corporal superior a $20 \%$ são considerados de alto risco nutricional.

Por outro lado, a presença de trauma metabólico (estresse), com produção aumentada de hormônios considerados, nestas situações, hipercatabólicos, também deve ser considerada. Uma vez considerado o estado geral do paciente, incluindo risco nutricional, a doença de base e estado hipercatabólico, deve ser iniciado o suporte nutricional. Nessas condições, a via parenteral deve ser utilizada sempre que for impossível se utilizar avia oral, fisiológica.

\section{PRESCRIÇÃO DE NU- TRIÇÃO PARENTERAL}

As células de todo organismo vivo necessitam de uma quantidade fixa diária de energia, para dos alimentos realizar suas reações metabólicas. Contudo, na incapacidade de medir-se o gasto energético por calorimetria indireta, calculam-se as necessidades energéticas de base, para o adulto, a partir de dados simples, porém aproximativos, por meio da equação de Harris \& Benedict, ou seja:

$\mathrm{E}=66,47+13,75 \times \mathrm{P}+5,00 \times \mathrm{A}-6,76 \times \mathrm{I}$ (homem)

$\mathrm{E}=655,09+9,56 \times \mathrm{P}+1,85 \times \mathrm{A}-4,68 \times \mathrm{I}$ (mulher)

onde:

$\mathrm{E}=$ Necessidades Energéticas de Base em kcal

$\mathrm{P}=$ Peso em $\mathrm{kg}$

$\mathrm{A}=$ Altura em $\mathrm{cm}$

$\mathrm{I}=$ Idade em anos

As necessidades calóricas de base, calculadas pela equação acima, situam-se entre 20 e 30 $\mathrm{kcal} . \mathrm{kg}^{-1} \mathrm{dia}^{-1}$. No entanto, em situações de estresse metabólico, como, por exemplo, na sepse, no pós-operatório ou no politraumatismo, ocorre um importante incremento nessas necessidades energéticas de base. Assim sendo, e considerando-se o trauma metabólico, propõe-se um acréscimo da oferta energética, como exposto a seguir ${ }^{(6)}$.

Acréscimo percentual aproximado, secundário a diferentes traumas metabólicos:

$\begin{array}{lr}\text { Cirurgia Eletiva } & 24 \\ \text { Fraturas } & 32 \\ \text { Traumatismo Craniano } & 61 \\ \text { Corticoterapia } & 61 \\ \text { Contusões } & 65 \\ \text { Infecção } & 70-79 \\ \text { Queimados } & 50 \text { a } 100\end{array}$

Tabela I - Indicações da nutrição parenteral total

- Impossibilidade do uso das vias oral/enteral

- Interferência de doença de base em ingestão, digestão ou a absorção

- Desnutrição com perda de massa corporal > 20\%

- Estados hipermetabólicos

- grandes queimados

- pacientes sépticos

- politraumatismo extenso

- pancreatite aguda

- fístulas intestinais de alto débito 
O cálculo calórico diário é, então, distribuído da seguinte maneira, de acordo com as necessidades energéticas, em macro (proteínas, lipídios e carboidratos) e micronutrientes (oligoelementos e vitaminas), ou seja: Proteínas

$0,8-1,0 \mathrm{~g} / \mathrm{kg}$ de peso corpóreo (até $2,0 \mathrm{~g} / \mathrm{kg}$ )

\section{Lipídios}

1,0 - 1,5 g/kg de peso corpóreo

Em geral, representam 20 a $40 \%$ da energia não protéica total.

\section{Hidratos de carbono}

4,0 - 5,0 g/kg de peso corpóreo, cujas necessidades individuais devem ser adaptadas de acordo com o caso clínico.

Em geral, representam 50 a $60 \%$ da energia não protéica total.

$\begin{array}{ll}\text { Ácido fólico } & 400 \mu \mathrm{g} \\ \text { Ácido pantotênico } & 15,0 \mathrm{mg} \\ \text { Biotina } & 60,0 \mathrm{mg} \\ \text { Cálcio } & 0,2-0,4 \mathrm{~g} \\ \text { Cloro } & 3-4 \mathrm{~g}(84-112 \mathrm{mEq}) \\ \text { Cobre } & 0,3-0,5 \mathrm{mg} \\ \text { Cromo } & 15-30 \mu \mathrm{g} \\ \text { Ferro } & 1-2 \mathrm{mg} \\ \text { Fósforo } & 0,4-0,8 \mathrm{~g} \\ \text { Iodo } & 0,15 \mathrm{mg} \\ \text { Magnésio } & 0,3 \mathrm{~g}(25 \mathrm{mEq}) \\ \text { Manganês } & 2-5 \mathrm{mg} \\ \text { Molibdênio } & 20-120 \mu \mathrm{g} \\ \text { Niacina } & 40,0 \mathrm{mg} \\ \text { Potássio } & 3-4 \mathrm{~g}(76-102 \mathrm{mEq}) \\ \text { Selênio } & 50-100 \mu \mathrm{g} \\ \text { Sódio } & 1-3 \mathrm{~g}(43-130 \mathrm{mEq}) \\ \text { Vitamina A } & 1000 \mu \mathrm{g} \\ \text { Vitamina B1 } & 3,0 \mathrm{mg} \\ \text { Vitamina B12 } & 5 \mu \mathrm{g} \\ \text { Vitamina B2 } & 3,6 \mathrm{mg} \\ \text { Vitamina B6 } & 4,0 \mathrm{mg} \\ \text { Vitamina C } & 100 \mathrm{mg} \\ \text { Vitamina D } & 5-10 \mu \mathrm{g} \\ \text { Vitamina E } & 10-15 \mathrm{mg} \\ \text { Vitamina K } & 200 \mu \mathrm{g} \\ \text { Zinco } & 3-12 \mathrm{mg} \\ & \end{array}$

\section{MON1TORIZAÇÃO DO PACIENTE, RECE- BENDO NUTRIÇÃO PARENTERAL}

A monitorização do suporte nutricional é feita com a utilização de dados clínicos e laboratoriais, (Tabela II). Os dados clínicos se relacionam com o bem estar geral, resposta ao tratamento da doença de base e a própria desnutrição. Nesta avaliação, estão incluídos aspectos gerais, de atividade, sinais vitais e relacionados ao balanço hídrico. Os dados laboratoriais incluem dosagens de eletrólitos e glicose.

\section{Tabela II - Monitorização de paciente submetido} à nutrição parenteral

Monitorização clínica:

- Aspectos gerais: sintomas que sugerem sobrecarga ou deficiência de líquidos, de glicose, de eletrólitos, etc

- Atividade física desenvolvida pelo paciente. Participação ativa no tratamento.

- Controle do peso e medidas antropométricas. Ba lanço hídrico

- Verificação da oferta de nutrientes.

- Cuidados gerais de controle de infecção.

Monitorização laboratorial:

- Glicemia

- No primeiro dia: duas a três vezes.

- Nos dias seguintes: uma vez ao dia. A seguir uma vez/semana.

- $\mathrm{Na} \mathrm{K} \mathrm{Ca} \mathrm{PO} \mathrm{Na} \mathrm{Mg}$ - Duas vezes por semana

\section{EXEMPLO DE SUPORTE NUTRICIONAL NO ADULTO}

Uma vez tendo sido indicado o suporte nutricional parenteral com base nas informações anteriores, sobre as necessidades básicas diárias de indivíduo adulto, propõe-se o seguinte e ciente do sexo masculino, com sessenta anos de idade, $1,60 \mathrm{~cm}$ de altura e $60 \mathrm{~kg}$ de peso, que, no quarto dia de pósoperatório de cirurgia abdominal pós-trauma aberto, apresentou um quadro clínico de sepse.

A base de cálculo das necessidades calóricas se faz, como foi descrito anteriormente, a partir da equação de Harris \& Benedict ${ }^{(5)}$ corrigida pelo trauma metabólico representado pela sepse ${ }^{(6)}$. Pode-se, então, definir o seguinte esquema nutricional para esse paciente: 
$\mathrm{E}=66,42+(13,75 \times 60)+(5 \times 160)-(6,77 \times 60)$

$\mathrm{E}=1285 \mathrm{kcal}$ (necessidades energéticas de repouso)

E total $=1285+0,79 \times 1285$ (onde o fator de trauma metabólico aplicado foi o de 79\%)

$\mathrm{E}$ total $=2300 \mathrm{kcal}$ (necessidades energéticas estimadas)

Assim, $38 \mathrm{kcal} \cdot \mathrm{kg}^{-1} \cdot \mathrm{dia}^{-1}(2300 \mathrm{kcal} / 60 \mathrm{~kg})$ cobrem as exigências metabólicas desse paciente. Em seguida, essa energia calculada deve ser distribuída entre os nutrientes, da seguinte maneira:

Proteína: 0,8 a $1,5 \mathrm{~g} / \mathrm{kg}$ de peso, ou seja, 48 a $90 \mathrm{~g} / \mathrm{dia}$ Lipídios: 1,0 a 1,5 g/kg de peso, ou seja, 60 a 90 g/dia, equivalente a 540 a $810 \mathrm{kcal}$.

Carboidratos: 4,0 a 5,0 g/kg, ou seja, 240 a 300 g/dia, equivalente a 960 a $1200 \mathrm{kcal}$.

Se esses cálculos forem determinados para nutrição parenteral, teremos:

- para as proteínas

- $1000 \mathrm{ml}$ de solução de aminoácidos a 10\% fornecem $100 \mathrm{~g}$ de proteínas (16 g de nitrogênio/L)

- para os lipídios

- 500 ml de solução de lipídios a 10\% fornecem 100 g de lipídios e, aproximadamente, $1000 \mathrm{kcal}$

- para os hidratos de carbono

- $500 \mathrm{ml}$ de soro glicosado a $50 \%$ fornecem $250 \mathrm{~g}$ de glicose e $1000 \mathrm{kcal}$.

A solução final será constituída de $2000 \mathrm{kcal}$ não protéicas em $2000 \mathrm{ml}$, com uma oferta protéica de $1,5 \mathrm{~g}$ de proteínas.kg'.dia* A forma de administração da Nutrição Parenteral calculada pode ser efetuada por meio de uma solução completa, conhecida com o nome de três em um, onde todos os macronutrientes estão presentes. Embora sejam essas as técnicas mais aprimoradas, em determinados centros, por motivos econômicos ou ainda por falta de tecnologia apropriada, torna-se difícil o emprego diário da solução de lipídios. Vários centros têm adotado a administração da solução de lipídios apenas duas vezes por semana, sem observarem o aparecimento da Síndrome de Deficiência de Ácidos Graxos Essenciais. Por essa razão, a Comissão de Nutrição Parenteral do Hospital das Clínicas de Ribeirão Preto decidiu optar pelo emprego de uma mistura nutritiva padrão, contendo aminoácidos e glicose (além de eletrólitos, oligoelementos e vitaminas), sendo a administração de lipídios limitada a duas ou três vezes por semana, dependendo do caso clínico.

\section{SUPORTE NUTRICIONAL NO PACIENTE PEDIÁTRICO}

Embora existam várias vantagens no emprego de soluções parenterais padronizadas, corno já foi citado, é importante ressaltar que há perda de especificidade para o paciente. Ressalta-se que nenhum regi me parenteral único pode sei- ideal para todos os pa cientes, com uma grande variedade de processos patológicos, nem para todas as idades, nem para o mesmo paciente durante todas as fases de sua doença ${ }^{(7)}$

Diferente do paciente adulto, a criança é um serem crescimento, com necessidades específicas para cada faixa etária: lactente, pré-escolar, escolar e adolescente. Acrescente-se a isso as particularidades dos RN pré-termo, principalmente aqueles de muito baixo peso, em relação ao balanço hídrico, tolerância à glicose, e necessidades de eletrólitos ${ }^{(8)}$. $\mathrm{O}$ volume hídrico de manutenção para uma criança prematura é maior que aquele de uma criança a termo: sua delicada epiderme, sua grande superfície corporal, relativa ao peso, e a pequena quantidade de gordura favorecem as perdas hídricas, acrescentando-se a isso o uso de encubadoras e fototerapia, tão necessárias nessa fase da vida.

Os prematuros apresentam baixa tolerância à glicose nos primeiros dias de vida e, para evitar os efeitos danosos da variação da osmolaridade sérica e a diurese osmótica, a infusão de glicose deve ser iniciada numa velocidade semelhante à taxa de produção hepática (6 rng/kg/min) com aumentos lentos e gradativos até atingir-se $11-12 \mathrm{mg} / \mathrm{kg} / \mathrm{min}$, ao redor de cinco a sete dias. Também há grande dificuldade no fornecimento de quantidades ideais de cálcio e fósforo. Os teores máximos desses elementos, que podem ser incorporados às soluções, são limitados pela sua solubilidade, determinada pelo $\mathrm{pH}$ das soluções, que, por sua vez, depende da concentração de aminoácidos e glicose presentes. Muitas vezes, não é possível infundir as necessidades preconizadas aos pequenos prematuros, principalmente quando é feita restrição hídrica ou quando parte do líquido é administrado através de medicação. Esse problema deixará de existir, quando houver disponibilidade no uso do glicerofosfato, que não sofre as influências descri tas acima ${ }^{(9,10)}$.

Considerando-se todas essas variáveis, apresentamos sete soluções disponíveis (P1 a P7), que serão testadas quanto a sua viabilidade e especificidade para a utilização nas crianças das várias faixas etárias e também nos prematuros de muito baixo peso. Em pe- 
diatria utilizamos as soluções três em um, onde to dos os elementos são infundidos diariamente, o que, além de permitir um melhor aproveitamento pelo organismo, necessita apenas de um acesso venoso, por vezes problemático nos pequenos pacientes. As soluções de P1 a P4 são dirigidas aos pré-termos e aos lactentes até $10 \mathrm{~kg}$. As soluções variam apenas nas concentrações de aminoácidos e lipídeos, e devem ser usadas de forma progressiva, a fim de se atingirem as necessidades ideais desses elementos, que seriam fornecidas com a solução $\mathrm{P} 4(2,5-3,0 \mathrm{~g} / \mathrm{kg}$ de aminoácidos e $3,0 \mathrm{~g} / \mathrm{kg}$ lipídeos). Infundindo-se aos lactentes ao redor de 100 a 120 m peso, as necessidades diárias de água, eletrólitos, micronutrientes e macronutrientes serão alcançadas ${ }^{(11,12)}$.

Para os prematuros, o volume de solução a ser infundida poderá ser maior, e, no caso de haver intole- rância à glicose, poderá ser acrescentada determina da quantidade de água a solução 1 mal, ao invés de se aumentar o volume da mesma. Para as crianças com peso de 10 a $40 \mathrm{~kg}$, estão estabelecidas as soluções de P5 a P7, também com diferenças apenas nas concentrações de aminoácidos e lipídios. Deve ser ressaltado que essas formulações cobrem as necessidades normais dos pacientes e qualquer suplementação terá que ser administrada à parte.

A Figura 1A representa o formulário de prescrição de nutrição parenteral para os pacientes pediátricos, contendo as soluções nutritivas parenterais padronizadas. a Figura $1 \mathrm{~B}$ representa o verso do formulário e a Figura IC contem os detalhes das formulações nutritivas padronizadas, bem como as necessidades diárias de água, calorias, micro e macronutrientes, e vitaminas as crianças das diversas faixas etárias.

\section{Soluções Padrão - Nutrições Parenterais Pediátricas}

\begin{tabular}{|l|c|c|c|c|c|c|c|c|}
\hline \multicolumn{1}{|c|}{ Componentes (mL) } & P1 & P2 & P3 & P4 & P5 & P6 & P7 & P8 \\
\hline Aminoácidos a 10\% (pediátrico) & 5,0 & 8,5 & 17,0 & 25,0 & 120,0 & 200,0 & 300,0 & \\
\hline Glicose 50\% & 15,0 & 15,0 & 20,0 & 25,0 & 160,0 & 240,0 & 300,0 & \\
\hline Emulsão lipídica 20\% & 2,0 & 4,2 & 8,5 & 12,5 & 60,0 & 100,0 & 150,0 & \\
\hline Cloreto de sódio a 20\% & 0,8 & 0,8 & 0,8 & 0,8 & 11,7 & 11,7 & 11,7 & \\
\hline Cloreto de potássio a 19,1\% & 0,4 & 0,4 & 0,4 & 0,4 & 7,8 & 7,8 & 7,8 & \\
\hline Gluconato de cálcio a 10\% & 3,9 & 3,9 & 3,9 & 3,9 & 39,0 & 39,0 & 39,0 & \\
\hline Sulfato de magnésio a 20\% & 0,1 & 0,1 & 0,1 & 0,1 & 2,1 & 2,1 & 2,1 & \\
\hline Fosfato monobásico de potássio 13,6\% & 1,0 & 1,0 & 1,0 & 1,0 & 12,0 & 12,0 & 12,0 & \\
\hline Oligoelementos (pediátrico) & 0,4 & 0,4 & 0,4 & 0,4 & 2,5 & 2,5 & 2,5 & \\
\hline Água destilada q.s.p. & 100,0 & 100,0 & 100,0 & 100,0 & 1000,0 & 1000,0 & 1000,0 & \\
\hline
\end{tabular}

REQUISIÇÃO DE NUTRIÇÃO PARENTERAL PEDIÁTRICA

Paciente
Velocidade de infusão
Médico Responsável/C.R.M.
Farmacêutico
INDICAÇÃo
P1 a P4: Recém-nascidos e crianças com até $10 \mathrm{~kg}$
P5 a P7: Crianças com mais de $10 \mathrm{~kg}$

Registro Total de frascos em $24 \mathrm{hs}$

Leito

Data

Assinale a formulação prescrita

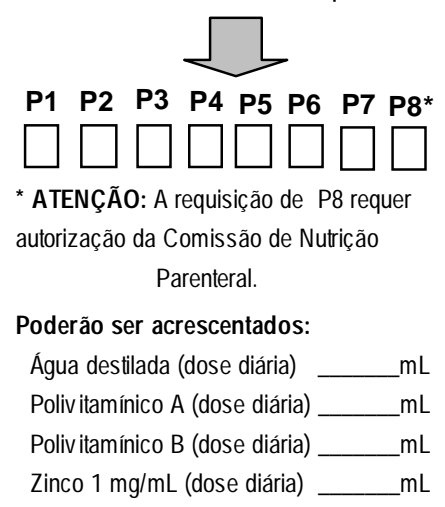

Figura $1 \mathrm{~A}$ - Formulário de prescrião parenteral para pacientes pediátricos internados no HCFMRP-USP, contendo Soluções Nutritivas Parenterais Padronizadas (Frente). 
Descrição das Dietas Parenterais Pediátricas Padronizadas

\begin{tabular}{|l|r|r|r|r|r|r|r|r|}
\hline \multicolumn{1}{|c|}{ Composição } & P1 & P2 & P3 & P4 & P5 & P6 & P7 & $\begin{array}{c}\text { P8 } \\
\text { (individ.) }\end{array}$ \\
\hline Aminoácidos a 10\% & $0,50 \mathrm{~g}$ & $0,85 \mathrm{~g}$ & $1,70 \mathrm{~g}$ & $2,50 \mathrm{~g}$ & $12,00 \mathrm{~g}$ & $20,00 \mathrm{~g}$ & $30,00 \mathrm{~g}$ & \\
\hline Glicose $50 \%$ & $7,50 \mathrm{~g}$ & $7,50 \mathrm{~g}$ & $10,00 \mathrm{~g}$ & $12,50 \mathrm{~g}$ & $80,00 \mathrm{~g}$ & $120,00 \mathrm{~g}$ & $150,00 \mathrm{~g}$ & \\
\hline Emulsão lipídica 20\% & $0,40 \mathrm{~g}$ & $0,84 \mathrm{~g}$ & $1,70 \mathrm{~g}$ & $2,50 \mathrm{~g}$ & $12,00 \mathrm{~g}$ & $20,00 \mathrm{~g}$ & $30,00 \mathrm{~g}$ & \\
\hline Sódio & $2,73 \mathrm{mEq}$ & $2,73 \mathrm{mEq}$ & $2,73 \mathrm{mEq}$ & $2,73 \mathrm{mEq}$ & $40,00 \mathrm{mEq}$ & $40,00 \mathrm{mEq}$ & $40,00 \mathrm{mEq}$ & \\
& $62,90 \mathrm{mg}$ & $62,90 \mathrm{mg}$ & $62,90 \mathrm{mg}$ & $62,90 \mathrm{mg}$ & $920,00 \mathrm{mg}$ & $920,00 \mathrm{mg}$ & $920,00 \mathrm{mg}$ & \\
\hline Potássio & $2,02 \mathrm{mEq}$ & $2,02 \mathrm{mEq}$ & $2,02 \mathrm{mEq}$ & $2,02 \mathrm{mEq}$ & $31,89 \mathrm{mEq}$ & $31,89 \mathrm{mEq}$ & $31,89 \mathrm{mEq}$ & \\
& $78,78 \mathrm{mg}$ & $78,78 \mathrm{mg}$ & $78,78 \mathrm{mg}$ & $78,78 \mathrm{mg}$ & $1243,71 \mathrm{mg}$ & $1243,71 \mathrm{mg}$ & $1243,71 \mathrm{mg}$ & \\
\hline Magnésio & $0,33 \mathrm{mEq}$ & $0,33 \mathrm{mEq}$ & $0,33 \mathrm{mEq}$ & $0,33 \mathrm{mEq}$ & $7,00 \mathrm{mEq}$ & $7,00 \mathrm{mEq}$ & $7,00 \mathrm{mEq}$ & \\
& $3,96 \mathrm{mg}$ & $3,96 \mathrm{mg}$ & $3,96 \mathrm{mg}$ & $3,96 \mathrm{mg}$ & $84,00 \mathrm{mg}$ & $84,00 \mathrm{mg}$ & $84,00 \mathrm{mg}$ & \\
\hline Cálcio & $1,81 \mathrm{mEq}$ & $1,81 \mathrm{mEq}$ & $1,81 \mathrm{mEq}$ & $1,81 \mathrm{mEq}$ & $18,14 \mathrm{mEq}$ & $18,14 \mathrm{mEq}$ & $18,14 \mathrm{mEq}$ & \\
& $36,20 \mathrm{mg}$ & $36,20 \mathrm{mg}$ & $36,20 \mathrm{mg}$ & $36,20 \mathrm{mg}$ & $362,80 \mathrm{mg}$ & $362,80 \mathrm{mg}$ & $362,80 \mathrm{mg}$ & \\
\hline Fósforo & $1,00 \mathrm{mmol}$ & $1,00 \mathrm{mmol}$ & $1,00 \mathrm{mmol}$ & $1,00 \mathrm{mmol}$ & $12,00 \mathrm{mmol}$ & $12,00 \mathrm{mmol}$ & $12,00 \mathrm{mmol}$ & \\
& $31,00 \mathrm{mg}$ & $31,00 \mathrm{mg}$ & $31,00 \mathrm{mg}$ & $31,00 \mathrm{mg}$ & $372,00 \mathrm{mg}$ & $372,00 \mathrm{mg}$ & $372,00 \mathrm{mg}$ & \\
\hline Cloreto & $3,75 \mathrm{mEq}$ & $3,75 \mathrm{mEq}$ & $3,75 \mathrm{mEq}$ & $3,75 \mathrm{mEq}$ & $60,00 \mathrm{mEq}$ & $60,00 \mathrm{mEq}$ & $60,00 \mathrm{mEq}$ & \\
& $133,12 \mathrm{mg}$ & $133,12 \mathrm{mg}$ & $133,12 \mathrm{mg}$ & $133,12 \mathrm{mg}$ & $2.130,00 \mathrm{mg}$ & $2.130,00 \mathrm{mg}$ & $2.130,00 \mathrm{mg}$ & \\
\hline Zinco & $200,00 \mathrm{mcg}$ & $200,00 \mathrm{mcg}$ & $200,00 \mathrm{mcg}$ & $200,00 \mathrm{mcg}$ & $1,500,00 \mathrm{mcg}$ & $1.500,00 \mathrm{mcg}$ & $1.500,00 \mathrm{mcg}$ & \\
\hline Cobre & $40,00 \mathrm{mcg}$ & $40,00 \mathrm{mcg}$ & $40,00 \mathrm{mcg}$ & $40,00 \mathrm{mcg}$ & $300,00 \mathrm{mcg}$ & $300,00 \mathrm{mcg}$ & $300,00 \mathrm{mcg}$ & \\
\hline Manganês & $4,00 \mathrm{mcg}$ & $4,00 \mathrm{mcg}$ & $4,00 \mathrm{mcg}$ & $4,00 \mathrm{mcg}$ & $30,00 \mathrm{mcg}$ & $30,00 \mathrm{mcg}$ & $30,00 \mathrm{mcg}$ & \\
\hline Cromo & $0,40 \mathrm{mcg}$ & $0,40 \mathrm{mcg}$ & $0,40 \mathrm{mcg}$ & $0,40 \mathrm{mcg}$ & $3,00 \mathrm{mcg}$ & $3,00 \mathrm{mcg}$ & $3,00 \mathrm{mcg}$ & \\
\hline Água destilada $(\mathrm{mL})$ & $66,20 \mathrm{~mL}$ & $66,20 \mathrm{~mL}$ & $48,90 \mathrm{~mL}$ & $24,90 \mathrm{~mL}$ & $489,40 \mathrm{~mL}$ & $314,40 \mathrm{~mL}$ & $104,40 \mathrm{~mL}$ & \\
\hline
\end{tabular}

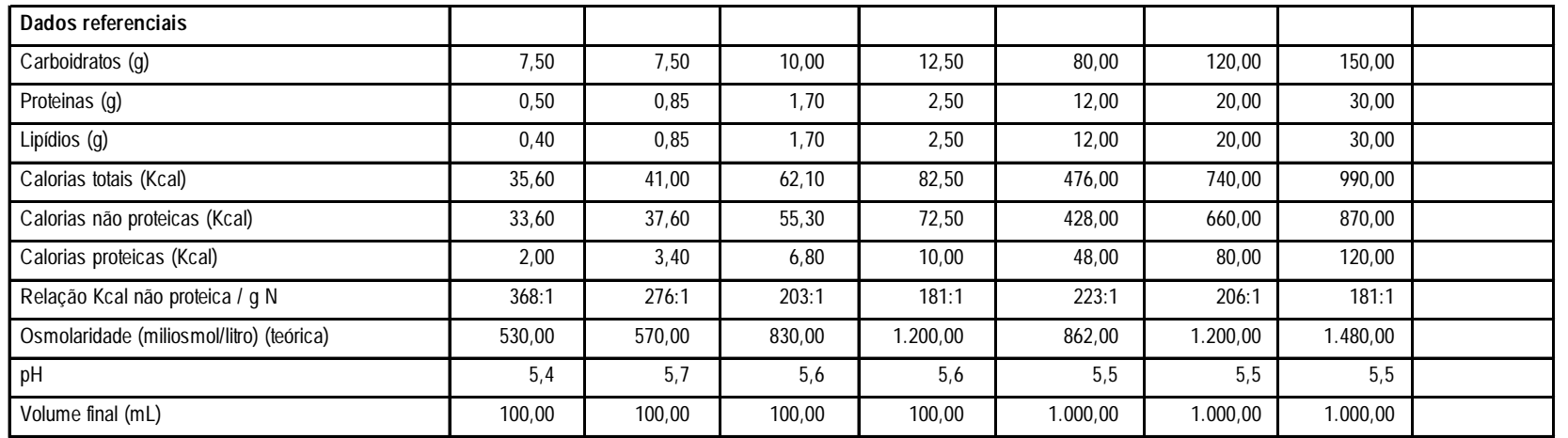

Figura 1 B - Formulário de prescrião parenteral para pacientes pediátricos internados no HCFMRP-USP, contendo Soluções Nutritivas Parenterais Padronizadas (verso). 


\begin{tabular}{|c|c|c|c|c|}
\hline \multirow[b]{2}{*}{ Elemento } & \multirow{2}{*}{$\begin{array}{l}\text { Pré-Termo } \\
\text { Neonatos } \\
(\mathrm{mcg} / \mathrm{kg})\end{array}$} & Termo & \multirow{2}{*}{$<5$ Anos } & \multirow[b]{2}{*}{$\begin{array}{c}\text { Crianças e } \\
\text { Adolescentes }\end{array}$} \\
\hline & & $\begin{array}{c}\text { Neonatos } \\
(\mathrm{mcg} / \mathrm{kg})\end{array}$ & & \\
\hline Zinco & 400 & 300 & 100 & $2-5 \mathrm{mg}$ \\
\hline Cobre & 20 & 20 & 20 & $200-500 \mathrm{mcg}$ \\
\hline Cromo & 0,2 & 0,2 & $0,14-0,2$ & $5-15 \mathrm{mcg}$ \\
\hline Manganês & 1 & 1 & $2-10$ & $50-150 \mathrm{mcg}$ \\
\hline
\end{tabular}

\begin{tabular}{lrrr}
\multicolumn{4}{c}{ DOSES DIÁRIAS RECOMENDADAS DE VITAMINAS } \\
& $\begin{array}{r}\text { < 1,5 kg } \\
\text { (kg/dia) }\end{array}$ & $\begin{array}{r}\text { Lactentes / } \\
\text { Crianças (dia) }\end{array}$ & Adultos (dia) \\
\hline A & $1.400 \mathrm{UI}$ & $2.300 \mathrm{UI}$ & $3.300 \mathrm{UI}$ \\
D & $240 \mathrm{UI}$ & $400 \mathrm{UI}$ & $200 \mathrm{UI}$ \\
E & $4,2 \mathrm{UI}$ & $7 \mathrm{UI}$ & $10 \mathrm{UI}$ \\
BI (Tiamina) & $072 \mathrm{mg}$ & $1,2 \mathrm{mg}$ & $3 \mathrm{mg}$ \\
B2 (Riboflavina) & $0,84 \mathrm{mg}$ & $1,4 \mathrm{mg}$ & $3,6 \mathrm{mg}$ \\
B3 (Niacina) & $10,2 \mathrm{mg}$ & $17,0 \mathrm{mg}$ & $10-150 \mathrm{mg}$ \\
B5 (Pantotênico) & $3,0 \mathrm{mg}$ & $5,0 \mathrm{mg}$ & $15,0 \mathrm{mg}$ \\
B6 (Piridoxina) & $0,6 \mathrm{mg}$ & $1,0 \mathrm{mg}$ & $4,0 \mathrm{mg}$ \\
B7 (Biotina) & $12,0 \mathrm{mcg}$ & $20,0 \mathrm{mcg}$ & $60,0 \mathrm{mcg}$ \\
B9 (Ácido fólico) & $84,0 \mathrm{mcg}$ & $140 \mathrm{mcg}$ & $400 \mathrm{mcg}$ \\
B12 (Cianocobalamina) & $0,6 \mathrm{mcg}$ & $1,0 \mathrm{mcg}$ & $5,0 \mathrm{mcg}$ \\
C (Ácido ascórbico) & $48 \mathrm{mg}$ & $80 \mathrm{mg}$ & $100 \mathrm{mg}$ \\
\hline
\end{tabular}

\begin{tabular}{ll}
$\begin{array}{c}\text { NECESSIDADES ENERGÉTICAS DIÁRIAS } \\
\text { (kcal não-protêica/kg) }\end{array}$ \\
\hline Neonatos pré-termo & $120-140$ \\
$<6$ meses & $90-120$ \\
$6-12$ meses & $80-100$ \\
$1-7$ anos & $75-90$ \\
$7-12$ anos & $60-75$ \\
$>12$ anos & $30-60$ \\
\hline
\end{tabular}

NECESSIDADES DIÁRIAS DE PROTEÍNAS (g/kg)

\begin{tabular}{lc}
\hline Neonatos & $2,5-3,0$ \\
Lactentes & $2,0-2,5$ \\
Crianças & $1,5-2,0$ \\
Adolescentes & $0,8-2,0$ \\
\hline
\end{tabular}

DOSES DIÁRIAS RECOMENDADAS DE ELETROLITOS

\begin{tabular}{lrrr} 
& Neonatos & Lactentes/Crianças & Adolescentes \\
\hline Sódio & $2-5 \mathrm{mEq} / \mathrm{kg}$ & $2-6 \mathrm{mEq} / \mathrm{kg}$ & $50-80 \mathrm{mEq}$ \\
Potássio & $1-4 \mathrm{mEq} / \mathrm{kg}$ & $2-3 \mathrm{mEq} / \mathrm{kg}$ & $40-60 \mathrm{mEq}$ \\
Cloreto & $1-5 \mathrm{mEq} / \mathrm{kg}$ & $2-5 \mathrm{mEq} / \mathrm{kg}$ & \\
Cálcio & $3-4 \mathrm{mEq} / \mathrm{kg}$ & $1-2,5 \mathrm{mEq} / \mathrm{kg}$ & $10-20 \mathrm{mEq}$ \\
Fósforo & $1-2 \mathrm{mmol} / \mathrm{kg}$ & $0,5-1 \mathrm{mmol} / \mathrm{kg}$ & $10-40 \mathrm{mEq}$ \\
Magnésio & $0,3-0,5 \mathrm{mEq} / \mathrm{kg}$ & $0,3-0,5 \mathrm{mEq} / \mathrm{kg}$ & $10-30 \mathrm{mEq}$ \\
\hline
\end{tabular}

NECESSIDADES DIÁRIAS DE LÍQUIDOS

\begin{tabular}{ll} 
Peso Corpóreo & Quantidade \\
\hline$<1.5 \mathrm{~kg}$ & $120-180 \mathrm{~mL} / \mathrm{kg}$ \\
$1,5-2,0 \mathrm{~kg}$ & $120-180 \mathrm{~mL} / \mathrm{kg}$ \\
$2,5-10 \mathrm{~kg}$ & $120 \mathrm{~mL} / \mathrm{kg}$ \\
$>10 \mathrm{~kg}-20 \mathrm{~kg}$ & $1.000 \mathrm{~mL}$ para $10 \mathrm{~kg} / 50 \mathrm{~mL} / \mathrm{kg}$ para cada $\mathrm{kg}>10$ \\
$>20 \mathrm{~kg}$ & $1.500 \mathrm{~mL}$ para $20 \mathrm{~kg} / 20 \mathrm{~mL} / \mathrm{kg}$ para cada $\mathrm{kg}>20$
\end{tabular}

SOLUÇÕES DE VITAMINAS UTILIZADAS EM NUTRIÇÃO PARENTERAL

Uso Pediátrico

POLIVIT PEDIÁTRICO A

Cada ampola de $\mathbf{1 0} \mathrm{ml}$ contém:

$\begin{array}{lr}\text { Vitamina A } & 2.300 \mathrm{UI} \\ \text { Vitamina D } & 400 \mathrm{UI} \\ \text { Vitamina E } & 7 \mathrm{UI} \\ \text { Vitamina B1 } & 1,2 \mathrm{mg} \\ \text { Vitamina B2 } & 1,4 \mathrm{mg} \\ \text { Vitamina B3 (Niacinamida) } & 17,0 \mathrm{mg} \\ \text { Vitamina B5 (Ácido pantotênico) } & 5,0 \mathrm{mg} \\ \text { Vitamina B6 } & 1,0 \mathrm{mg} \\ \text { Vitamina C } & 80,0 \mathrm{mg}\end{array}$

POLIVIT PEDIÁTRICO B

Cada ampola de $5 \mathrm{ml}$ contém:

Vitamina B7 (Biotina) $20 \mathrm{mcg}$

Vitamina B9 (Ácido fólico) $\quad 140 \mathrm{mcg}$

Vitamina B12 $1 \mathrm{mcg}$

NOTA: Ampola B complementa a ampola A

Figura 1C - Recomendações diárias em micro e macronutrientes em diferentes idades.

\section{PADRONIZAÇÃO DOS NUTRIENTES: Melhor Controle nos Gastos}

Uma das grandes metas a ser atingida, pelas Comissões Multidisciplinares de Suporte Nutricional, é assegurar aos pacientes hospitalizados a assistência nutricional adequada, a fim de concentrar esforços na redução da morbidade e mortalidade causadas pela desnutrição e, dessa maneira, melhorar o prognóstico geral desses pacientes ${ }^{\circ}$ tornando o custo benefício procedimento o mais adequado possível Entre as várias medidas que podem ser adotadas, a mais importante é a padronização das soluções nutritivas parenterais.

No caso específico das proteínas, por exemplo, a padronização é feita através dos três tipos de soluções de aminoácidos disponíveis em nosso meio. Assim, dispõe-se de soluções de aminoácidos totais (uso genérico), de aminoácidos essenciais com histidina (para pacientes nefropatas) e de aminoácidos de ca- 
deia ramificada (para pacientes hepatopatas). A partir desses três tipos de solução de aminoácidos, adicionam-se hidratos de carbono, eletrólitos, oligoelementos e vitaminas, de acordo com as recomendações diárias para os pacientes adultos ou pediátricos, preconizadas pelas organizações internacionais, que estudam as necessidades básicas diárias em micro e macronutrientes Enfim, dois frascos da solução nutritiva parenteral determinada correspondem, aproximadamente, às necessidades caloriconitrogenadas dos pacientes hospitalizados. Além disso, um esquema complementar, como, por exemplo, a vitamina $\mathrm{K}$ uma ou duas vezes por semana, é seguido para completar as demandas metabólicas desses pacientes. As Figuras 1A-lB e 2A-2B correspondem aos formulários, frente e verso, adotados pela Comissão de Nutrição Parenteral do Hospital das Clínicas de Ribeirão Preto, para a prescrição de nutrição parenteral, em pacientes pediátricos e adultos, respectivamente.

Em conclusão, acredita-se que as vantagens de formação da Comissão de Nutrição Parenteral, com a respectiva padronização das soluções nutritivas parenterais, sejam eficazes por várias razões, entre elas as citadas a seguir:

1. evita-se o desperdício, por erros de cálculo, das necessidades diárias de cada paciente;

2. diminui-se a quantidade de manipulações de soluções nutritivas parenterais, efetuadas pelo Serviço de Farmácia, que prepara as soluções padronizadas apenas uma vez por dia;

3. incrementa-se o controle de qualidade dos nutrientes administrados;

4. as soluções nutritivas parenterais padronizadas cor respondem às necessidades metabólicas de quase to dos os pacientes hospitalizados;

5. controla-se, de maneira mais eficaz, o consumo e, sobretudo, os gastos com suporte nutricional parenteral.

6. os efeitos colaterais, resultantes do uso inadvertido de nutrientes potencialmente nocivos, como, por exemplo, excesso de potássio, são praticamente abolidos.

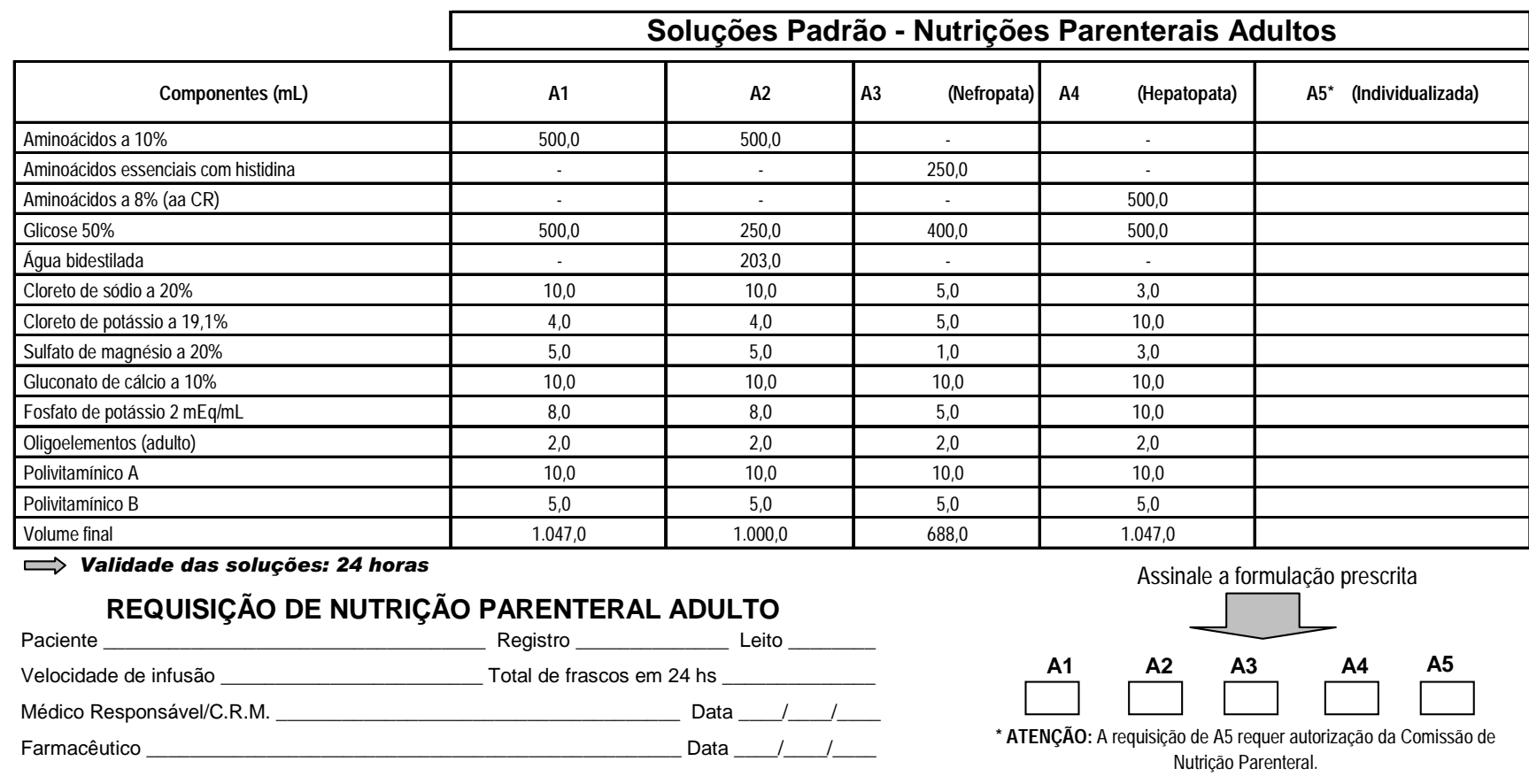

Figura 2 A - Formulário de prescrião parenteral para pacientes adultos, internados no HCFMRP-USP, contendo Soluções Nutritivas Parenterais Padronizadas (frente). 
Descrição das Dietas Parenterais Padronizadas

\begin{tabular}{|c|c|c|c|c|c|}
\hline Composição & A1 & A2 & $\begin{array}{c}\text { A3 } \\
\text { (Nefropata) } \\
\end{array}$ & $\begin{array}{c}\text { A4 } \\
\text { (Hepatopata) } \\
\end{array}$ & $\begin{array}{c}\text { A5 } \\
\text { (Individualizada) }\end{array}$ \\
\hline Aminoácidos a $10 \%$ & $50,00 \quad \mathrm{~g}$ & $50,00 \quad \mathrm{~g}$ & - & - & \\
\hline Aminoácidos essenciais $6,9 \%$ com histidina & - & - & $16,75 \mathrm{~g}$ & - & \\
\hline Aminoácidos a 8\% (aa CR) & - & - & - & $40,00 \mathrm{~g}$ & \\
\hline Glicose $50 \%$ & $250,00 \mathrm{~g}$ & $125,00 \mathrm{~g}$ & $200,00 \quad \mathrm{~g}$ & $250,00 \quad \mathrm{~g}$ & \\
\hline \multirow[t]{2}{*}{ Sódio } & $34,18 \mathrm{mEq}$ & $34,18 \mathrm{mEq}$ & $17,09 \mathrm{mEq}$ & $10,25 \mathrm{mEq}$ & \\
\hline & $786,14 \mathrm{mg}$ & $786,14 \mathrm{mg}$ & $393,07 \mathrm{mg}$ & $235,75 \mathrm{mg}$ & \\
\hline \multirow[t]{2}{*}{ Potássio } & $26,25 \mathrm{mEq}$ & $26,25 \mathrm{mEq}$ & $22,81 \mathrm{mEq}$ & $45,63 \mathrm{mEq}$ & \\
\hline & $1.023,75 \mathrm{mg}$ & $1.023,75 \mathrm{mg}$ & $889,68 \mathrm{mg}$ & $1.779,57 \mathrm{mg}$ & \\
\hline \multirow[t]{2}{*}{ Magnésio } & $16,66 \mathrm{mEq}$ & $16,66 \mathrm{mEq}$ & $3,33 \mathrm{mEq}$ & $10,00 \mathrm{mEq}$ & \\
\hline & $199,92 \mathrm{mg}$ & $199,92 \mathrm{mg}$ & $39,96 \mathrm{mg}$ & $120,00 \mathrm{mg}$ & \\
\hline \multirow[t]{2}{*}{ Cálcio } & $4,65 \mathrm{mEq}$ & $4,65 \mathrm{mEq}$ & $4,65 \mathrm{mEq}$ & $4,65 \mathrm{mEq}$ & \\
\hline & $93,00 \mathrm{mg}$ & $93,00 \mathrm{mg}$ & $93,00 \mathrm{mg}$ & $93,00 \mathrm{mg}$ & \\
\hline \multirow[t]{2}{*}{ Fósforo } & $44,00 \mathrm{mEq}$ & $44,00 \mathrm{mEq}$ & $27,50 \mathrm{mEq}$ & $55,00 \mathrm{mEq}$ & \\
\hline & $272,80 \mathrm{mg}$ & $272,80 \mathrm{mg}$ & $170,50 \mathrm{mg}$ & $341,00 \mathrm{mg}$ & \\
\hline Cloreto & $44,43 m E q$ & $44,43 \mathrm{mEq}$ & $29,90 \mathrm{mEq}$ & $35,88 \mathrm{mEq}$ & \\
\hline & $2.130,00 \mathrm{mg}$ & $2.130,00 \mathrm{mg}$ & $1.065,00 \mathrm{mg}$ & $1.960,00 \mathrm{mg}$ & \\
\hline Vitamina A (Retinol) & $3.300,00 \mathrm{UI}$ & $3.300,00 \mathrm{UI}$ & $3.300,00 \quad \mathrm{UI}$ & $3.300,00 \quad \mathrm{UI}$ & \\
\hline Vitamina B1 (Tiamina) & $3,00 \mathrm{mg}$ & $3,00 \mathrm{mg}$ & $3,00 \mathrm{mg}$ & $3,00 \mathrm{mg}$ & \\
\hline Vitamina B2 (Riboflavina) & $3,60 \mathrm{mg}$ & $3,60 \mathrm{mg}$ & $3,60 \mathrm{mg}$ & $3,60 \mathrm{mg}$ & \\
\hline Vitamina B3 (Nicotinamida) & $40,00 \mathrm{mg}$ & $40,00 \mathrm{mg}$ & $40,00 \mathrm{mg}$ & $40,00 \mathrm{mg}$ & \\
\hline Vitamina B5 (Dexpantenol) & $15,00 \mathrm{mg}$ & $15,00 \mathrm{mg}$ & $15,00 \mathrm{mg}$ & $15,00 \mathrm{mg}$ & \\
\hline Vitamina B6 (Piridoxina) & $4,00 \mathrm{mg}$ & $4,00 \mathrm{mg}$ & $4,00 \mathrm{mg}$ & $4,00 \mathrm{mg}$ & \\
\hline Vitamina C (Ácido ascórbico) & $100,00 \mathrm{mg}$ & $100,00 \mathrm{mg}$ & $100,00 \mathrm{mg}$ & $100,00 \mathrm{mg}$ & \\
\hline Vitamina D (Ergocalciferol) & $200,00 \mathrm{UI}$ & $200,00 \mathrm{UI}$ & $200,00 \mathrm{UI}$ & $200,00 \mathrm{UI}$ & \\
\hline Vitamina $\mathrm{E}$ (Tocoferol) & $10,00 \mathrm{UI}$ & $10,00 \mathrm{UI}$ & $10,00 \mathrm{UI}$ & $10,00 \mathrm{UI}$ & \\
\hline Vitamina B7 (Biotina) & $0,03 \mathrm{mg}$ & $0,03 \mathrm{mg}$ & $0,03 \mathrm{mg}$ & $0,03 \mathrm{mg}$ & \\
\hline Vitamina B9 (Ácido fólico) & $0,20 \mathrm{mg}$ & $0,20 \mathrm{mg}$ & $0,20 \mathrm{mg}$ & $0,20 \mathrm{mg}$ & \\
\hline Vitamina B12 (Cianocobalamina) & $3,00 \mathrm{mcg}$ & $3,00 \mathrm{mcg}$ & $3,00 \mathrm{mcg}$ & $3,00 \mathrm{mcg}$ & \\
\hline Zinco & $5,00 \mathrm{mg}$ & $5,00 \mathrm{mg}$ & $5,00 \mathrm{mg}$ & $5,00 \mathrm{mg}$ & \\
\hline Cobre & $1,60 \mathrm{mg}$ & $1,60 \mathrm{mg}$ & $1,60 \mathrm{mg}$ & $1,60 \mathrm{mg}$ & \\
\hline Manganês & $0,80 \mathrm{mg}$ & $0,80 \mathrm{mg}$ & $0,80 \mathrm{mg}$ & $0,80 \mathrm{mg}$ & \\
\hline Cromo & $0,02 \mathrm{mg}$ & $0,02 \mathrm{mg}$ & $0,02 \mathrm{mg}$ & $0,02 \mathrm{mg}$ & \\
\hline Água destilada (mL) & - & $203,00 \mathrm{ml}$ & - & - & \\
\hline Dados referenciais & & & & & \\
\hline Carboidratos (g) & 250,00 & 125,00 & 200,00 & 250,00 & \\
\hline Proteinas (g) & 50,00 & 50,00 & 16,75 & 40,00 & \\
\hline Calorias totais (Kcal) & $1.200,00$ & 700,00 & 867,00 & $1.160,00$ & \\
\hline Calorias não protéicas (Kcal) & $1.000,00$ & 500,00 & 800,00 & $1.000,00$ & \\
\hline Calorias protéicas (Kcal) & 200,00 & 200,00 & 67,00 & 160,00 & \\
\hline Relação Kcal não protéica / g N & $125: 1$ & $63: 1$ & $298: 1$ & $156: 1$ & \\
\hline Osmolaridade (miliosmol/litro) (teórica) & $2.103,00$ & $1.357,00$ & $1.474,00$ & $1.930,00$ & \\
\hline $\mathrm{pH}$ & 6,2 & 5,9 & 5,7 & 5,2 & \\
\hline Volume final $(\mathrm{mL})$ & $1.047,00$ & $1.000,00$ & 688,00 & $1.047,00$ & \\
\hline
\end{tabular}

Figura 2B. Formulário de prescrição de nutrição parenteral para pacientes adultos internados no Hospital das Clínicas de Ribeirão Preto da Faculdade de Medicina de Ribeirão Preto (VERSO). 
DOSES DIÁRIAS RECOMENDADAS DE ELETRÓLITOS

\begin{tabular}{lr}
\hline Sódio & $50-200 \mathrm{mEq}$ \\
Potássio & $30-100 \mathrm{mEq}$ \\
Cloreto & $50-200 \mathrm{mEq}$ \\
Cálcio & $3-30 \mathrm{mEq}$ \\
Fósforo & $10-40 \mathrm{mMol}$ \\
Magnésio & $10-30 \mathrm{mEq}$
\end{tabular}

DOSES DIÁRIAS RECOMENDADAS DE OLIGOELEMENTOS

$\begin{array}{ll}1 \text { - ZINCO } & 2,5 \text { a } 5,0 \mathrm{mg} \\ \text { 2 - COBRE } & 0,5 \text { a } 1,5 \mathrm{mg} \\ \text { 3 - CROMO } & 10 \text { a } 15 \mathrm{mcg} \\ \text { 4 - MANGANÊS } & 0,15 \text { a } 0,8 \mathrm{mg}\end{array}$

DOSES DIÁRIAS RECOMENDADAS
$\begin{array}{ll}\text { DE VITAMINAS SEGUNDO A AMA/FDA } \\ \text { A } & 3.300 \mathrm{UI} \\ \text { D } & 200 \mathrm{UI} \\ \text { E } & 10 \mathrm{UI} \\ \text { B1 (Tiamina) } & 3 \mathrm{mg} \\ \text { B2 (Riboflavina) } & 3,6 \mathrm{mg} \\ \text { B3 (Niacina) } & 40 \mathrm{mg} \\ \text { B5 (Pantotênico) } & 15 \mathrm{mg} \\ \text { B6 (Piridoxina) } & 4 \mathrm{mg} \\ \text { B7 (Biotina) } & 60 \mathrm{mcg} \\ \text { B9 (Ácido fólico) } & 400 \mathrm{mcg} \\ \text { B12 (Cianocobalamina) } & 5 \mathrm{mcg} \\ \text { C (Ácido ascórbico) } & 100 \mathrm{mg} \\ \text { K } & 5 \mathrm{mg} / \mathrm{semana}\end{array}$

Figura 2C - Recomendações diárias de micro e macronutrientes para o adulto

MARCHINI JS et al. Parenteral nutrition support - prescription and follow up. Medicina, Ribeirão Preto, 31: 62-72, Jan. /March 1998.

ABSTRACT: The introduction of total parenteral nutrition support in the therapy of hospitalized patients has necessitated the development of a rig guidelines lo promote coast containment and optimal patient care. It is very kwon nowadays the impact of nutrition support team, which members include one or more physicians, nurses and pharmacists upon this practice, such as, the potential benefits derived from a particular mode of providing specifics parenteral feeding formulations and high performance in the assessment of nutrient intake of the hospitalized patient. The main purpose of this study is to present the Total Parenteral Nutrition Committee of the Clinics Hospital of Ribeirão Preto's report concerning the specific parenteral feeding formulations to be used in the hospitalized patient.

UNITERMS: Parenteral Nutrition, Total. Formularies. Committee.

\section{REFERENCIAS BIBLIOGRAFICAS}

1 - DUDRICK SJ et al. Long-term parenteral nutrition with growth, development and positive nitrogen balance. Surgery 64: 134142, 1968.

2- BUTTERWORTH Jr CE. Editorial: Malnutrition in the hospital. JAMA 230: 879, 1974.

3 - BLACKBURN GL et al. Nutritional and metabolic assess mentol the hospitalized patient. JPEN J Parenter Enteral Nutr 1:1122, 1977.
4- BASILE FILHO A et al. A importância da formação de equipe multidisciplinar de suporte nutricional sobre a economia hospitalar. Rev Fac Cien Med Univ Campinas 1: 47-54, 1989.

5- HARRIS JA \& DENEDICT FO. A biometric study of basal metabolism in man. Carnegie Institute of Washington, Washington, D.C., Public. n 279, 1919.

6 - LONG CL et al. Metabolic response to injury and illness: Estimation of energy protein needs from indirect calorimetry and nitrogen balance. JPEN J Parenter Enteral Nutr 3: 452456, 1979. 
7 - GRACEY M \& MacMAHON RA. Enteral and parenteral nutrition. in: GRACEY M \& BURKE V, eds. Pediatric gastroenterology and hepatology, 3 th Ed, Blackwell Scientific Publication, Boston, p. 924-938, 1993.

8 - HEIRD WC; KASHYAP S \& GOMEZ MR. Parenteral alimentation of the neonate. Semin Perinatol 15: 493-502, 1991.

9- KERNER Jr JA. Manual of pediatric parenteral nutrition. Wiley Medical Publication, New York, 1983. 365p.

10 - KERNER Jr JA. Parenteral nutrition. In: WALKER W et al. eds. Pediatric gastrointestinal disease: pathophysiology, diagnosis, management. B.C. Decken, Philadelphia, p. 1645—1672, 1991.

11 - PEREIRA GR. Nutritional care of the extremely premature infant. Clin Perinatol 22: 61-75, 1995.

12 - SHULMAN RJ. Nutrição parenteral em lactentes e crianças. In: CARRAZA FR \& MARCONDES E. ed. Nutrição clínica em pediatria. Sarvier, São Paulo, p. 288-302, 1991.
13 - TRAEGER SM et al. Total parenteral nutrition by a nutritional support team: improved quality of care. JPEN J Parenter Enteral Nutr 10: 408-412, 1986.

14- O'BRIEN DD et al. Recommendations of Nutritional supporttion. Team promote cost containment. JPEN J Parenter Enteral Nutr 10: 300-302, 1986.

15 - FRIEDMAND MH; HIGA AM \& DAVIS AJ. A unique team approach to optimal nutrition support whit minimal coat. Nutr Supp Services 3: 27-28, 1983.

16- COMMITTEE ON DIETARY ALLOWANCES; Committee on interpretation of the Recommended Dietary Allowances. Recommended Dietary Allowances. 10 th ed., National Academy of Sciences, Washington, D.C., 1985.

Recebido para publicação em 30/01/98

Aprovado para publicação em 25/02/98 